\title{
Acute myelomonocytic leukaemia complicated by an acute aortic thrombosis
}

\author{
KW Leong, JJ Bosco, IB Shaik
}

\begin{abstract}
Summary
Acute aortic thrombosis is a rare condition, occurring mainly as a result of trauma or atherosclerosis and occasionally secondary to hypercoagulable states. We report a patient with relapsed acute myeloid leukaemia who developed an unusual complication, acute aortic thrombosis.
\end{abstract}

Keywords: acute myeloid leukaemia, lupus anticoagulant, acute aortic thrombosis

\section{Introduction}

Acute aortic thrombosis is relatively rare (see box).$^{1-4} \mathrm{We}$ report a patient with relapsed acute myeloid leukaemia who developed an acute aortic thrombosis.

\section{Case report}

$\mathrm{KJ}$, a 38-year-old Malay man, presented as an acute emergency when he spontaneously developed sudden pain and numbness over his lower limbs with weakness, progressing to complete paralysis within one hour. $\mathrm{He}$ had urinary retention. One day earlier, he had had numbness and pain over his lower limbs lasting 15 minutes without any weakness. This resolved spontaneously.

He had been treated for acute myelomonocytic leukaemia a year earlier. He achieved remission after two induction courses consisting of daunorubicin and cytosine arabinoside. This was consolidated with two courses of chemotherapy consisting of VP 16 and cytosine arabinoside. He relapsed six weeks prior to the current presentation. He had refused reinduction with chemotherapy.

Faculty of Medicine, University of Malaya, Lembah Pantai, 59100 Kuala Lumpur,

Malaya

Department of

Medicine

$\mathrm{KW}$ Leong

JJ Bosco

Department of

Radiology

IB Shaik

Accepted 11 October 1994
On examination, he was in severe pain. Marked pallor with gross gum hypertrophy was present. He did not have any petechial haemorrhages. Blood pressure was 190/ $100 \mathrm{mmHg}$. Both lower limbs were mottled and cold. The femoral and all lower limb pulses were absent. He had Grade 0 power in both lower limbs. Reflexes were absent. A sensory level at T10 was present. No abdominal bruit was heard. Bowel sounds were present. No fundal haemorrhages were seen. There was no lymphadenopathy or hepatosplenomegaly.

A full blood count showed a haemoglobulin level of $5.3 \mathrm{~g} / \mathrm{dl}$, platelet count $84 \times 10^{9} / 1$, white blood cells $50.4 \times 10^{9} / 1$ with $60 \%$ blasts.

A dynamic CT scan of the abdomen revealed an aortic thrombus beginning just below the renal arteries and progressing down to both common iliac arteries (figure). The thrombus had almost occluded the whole lumen of the aorta. The para-aortic lymph nodes were normal and no abnormal masses were seen. Echocardiography was normal

The lupus anticoagulant was detected using the kaolin clotting time test and the tissue thromboplastin index. Antithrombin III and proteins $\mathrm{C}$ and $\mathrm{S}$ were found to be normal. Antiphospholipid antibody testing was not performed (table).

The perfusion of his lower limbs improved with heparin infusion. The mottled appearance had disappeared six hours later and his limbs were warmer. He regained grade 1 power to his

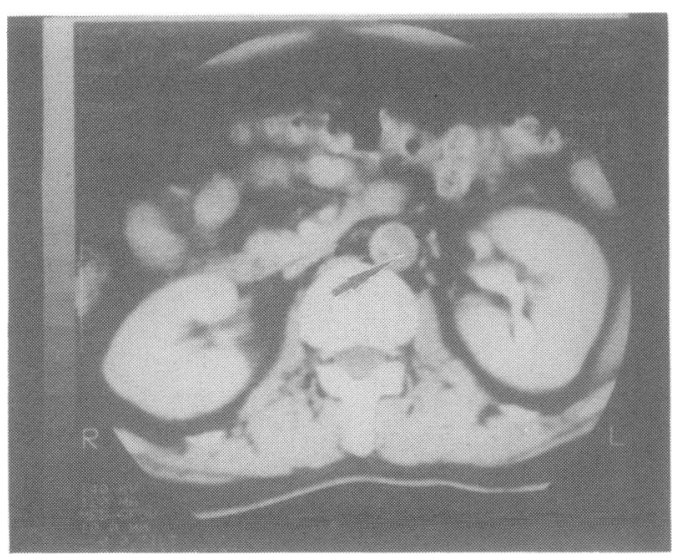

Figure Dynamic CT scan image of the abdomen just below the renal arteries demonstrating an irregular hypodense filling defect consistent with a thrombus within the aorta, occupying $75 \%$ of the lumen (arrow) The aortic diameter is normal 


\begin{tabular}{|l|}
\hline Acute aortic thrombosis: features \\
\hline - sudden lower limb ischaemia \\
- sudden hypertension \\
\hline
\end{tabular}

lower limbs and his sensory level improved to L3 level. All pulses in his lower limbs remained impalpable. His blood pressure returned to $150 / 85 \mathrm{mmHg}$ after a day. He was able to micturate and also had normal bowel opening by the third admission day. He was subsequently started on warfarin. A repeat CT scan six days later did not show any improvement nor any progression of the thrombosis.

Unfortunately, he discharged himself against medical advice 14 days after admission when he was improving and pain free. He did not wish to have any further treatment for his relapsed acute myelomonocytic leukaemia. $\mathrm{He}$ passed away at home 10 days after discharge.

\section{Discussion}

The patient had developed acute aortic thrombosis following the relapse of acute myeloid leukaemia. He had no known risk factors for an acute thrombosis except possibly the lupus anticoagulant. Acute leukaemia should predispose him to bleeding rather than thrombosis.

Acute aortic thrombosis is an uncommon problem. The classical presentation is sudden symptoms and signs of lower limb ischaemia, which may mimic post-mortem changes. ${ }^{1} \mathrm{~A}$ proportion of patients develops sudden hypertension. ${ }^{5}$ This may be due to renal artery occlusion or increase in peripheral vascular resistance. The blood pressure in the patient was only transiently high, which might have been partly contributed by severe pain. The important feature is impalpable pulses in the lower limbs. In patients with infrarenal aortic thrombosis, ${ }^{6}$ the arterial supply to the spinal cord is preserved. The neurological deficit is caused by ischaemic peripheral neuropathy causing sudden extreme pain in the lower limbs followed by paraesthesia. This appears to have been the case in this patient. Mesenteric infarction can occur if the inferior mesenteric artery is involved.

1 Babu SC, Shah PM, Sharma P, Patel KR, Clauss RH. Adequacy of central haemodynamics versus restoration of circulation in survival of patients with acute aortic thrombosis. Am f Surg 1987; 154: 206-10.

2 Shapiro ME, Rodvein R, Bauer KA, Salzman EW. Acute aortic thrombosis in antithrombin III deficiency. $\mathcal{f} A M A$ 1981; 245: 1759-61.

3 Gremse DA, Peery KJ, Walterspiel JN, Johnson WH Jr. Aortic thrombosis in a neonate with undetectable protein C. Pediat Radiol 1990; 20: 198-9.
Table Results of the lupus anticoagulant test

\begin{tabular}{lc}
\hline Test & Result \\
\hline Kaolin clotting time & \\
Normal plasma & $61.0 \mathrm{~s}$ \\
Normal plasma + patient plasma & $72.2 \mathrm{~s}$ \\
Patient plasma & $146.3 \mathrm{~s}$ \\
Index & $11.0 \mathrm{~s}$ \\
Platelet neutralising procedure & \\
Patient's plasma + freeze-thawed platelets & $57.0 \mathrm{~s}$ \\
Patient's plasma + TRIS buffered saline & $56.9 \mathrm{~s}$ \\
Tissue thromboplastin index & \\
1/100 & 1.9 \\
1/1000 & 2.2 \\
Normal ratio $\leqslant 1.3$ & \\
Russell viper venon & \\
Normal plasma & $23.2 \mathrm{~s}$ \\
Patient's plasma & $21.7 \mathrm{~s}$ \\
Ratio & 0.93 \\
Normal ratio & $<1.2$ \\
\hline
\end{tabular}

Two tests for the lupus anticoagulant were positive. This may explain the thrombotic tendency in this patient. There is one previous report of a case of aortic thrombosis associated with the antiphospholipid antibody ${ }^{4}$ It is possible that immunological perturbations in leukaemia had contributed somehow to the development of the lupus anticoagulant or some other procoagulant leading to aortic thrombosis. A white blood cell thrombus may be another explanation but the total white cell count was only $56 \times 10^{9} / 1$.

The treatment of choice for acute aortic thrombosis is emergency surgery with aortic reconstruction or a bypass procedure that carries a mortality of $60 \%{ }^{1,6}$ It would probably have been futile in this patient, who had thrombocytopenia and relapsed acute myeloid leukaemia. Thus, anticoagulation with heparin and warfarin was used. Fibrinolytic agents like streptokinase or urokinase would have been considered but the risks of a major haemorrhage with thrombocytopenia precluded their use. We are unaware of any report of its use in this situation. The improvement in circulation was probably due to restoration by collaterals and flow through an extremely narrowed aortic lumen.

We would like to thank Prof N Menaka, Dr Lim Ee Jin and Ms PL Koong for their help with the coagulation tests and the lupus anticoagulant testing.

4 McGee GS, Pearce WH, Sharma L, Green D, Yao JST Antiphospholipid antibodies and arterial thrombosis. Arch Surg 1992; 127: 342-6.

5 Wyatt GM, Felson B. Aortic thrombosis as a cause of hypertension: an arteriographic study. Radiology 1957; 69: 676-82.

6 McCullough JL Jr, Mackey WC, O'Donnell TF Jr, Millan VG, Deterling RA Jr, Callow AD. Infrarenal aortic occlusion: a reassessment of surgical indications. Am $\mathcal{f}$ Surg 1983; 146: 178-82. 